\title{
Soft Microscopy Of Macromolecules: Correlative Imaging and Enhancing Contrast
}

\author{
Kelly A Parker ${ }^{1}$, Sharan Bobbala ${ }^{2}$, Irina V Drachuk ${ }^{3}$, Peter A Mirau ${ }^{4}$, Jorge L Chavez ${ }^{3}$, Lawrence F \\ Drummy ${ }^{4}$, Evan A Scott ${ }^{2}$, and Vinayak P Dravid ${ }^{1,5 *}$ \\ 1. Department of Materials Science \& Engineering, Northwestern University, Evanston, IL, USA \\ 2. Department of Biomedical Engineering, Northwestern University, Evanston, IL, USA \\ 3. 711 Human Performance Wing, Air Force Research Laboratory, Dayton, OH, USA \\ 4. Materials and Manufacturing Directorate, Air Force Research Laboratory, Dayton, OH, USA \\ ${ }^{5 .}$ NUANCE Center, Northwestern University, Evanston, IL, USA \\ * Corresponding author: v-dravid@northwestern.edu
}

The principal challenge in soft microscopy is two-fold: biological materials have inherently low contrast, and their structures are altered by beam damage at electron doses that would improve this contrast. It is therefore necessary to both enhance contrast and reduce beam damage by a number of possible methods, beginning with specimen preparation, then optimizing imaging conditions through voltage and dose considerations, and finally by leveraging correlative techniques that provide complementary information. We address these challenges through the visualization of two model systems using transmission electron microscopy (TEM) and scanning TEM (STEM). DNA combing, pioneered by Bensimon et al. [1], produces elongated and aligned strands of known length that can be used to validate novel sample preparation and correlative imaging techniques, which can then be further implemented in gene sequencing and other applications. Bicontinuous nanospheres (BCNs) [2] contain twisted hydrophobic bilayers and aqueous internal channels, and are used to demonstrate targeted staining of nanoparticle systems for the improved contrast of specific macromolecules.

DNA was combed on an amorphous carbon film following a procedure similar to Nakao et al. [3]; 7,000 base pair DNA was deposited on a hydrophobic carbon-coated TEM grid, and the droplet was removed by pipette suction to induce DNA elongation. The grid was then hydrophilized and stained with $1 \%$ uranyl acetate. BCNs were synthesized from the diblock copolymer poly(ethylene glycol) 17 -blockpoly(propylene sulfide) 75 (PEG-PPS) by flash nanoprecipitation as described by Bobbala et al. [2] and loaded with 50 base pair double stranded DNA. BCNs were selectively stained for DNA using an adaption of the chromatin electron microscopy (ChromEM) method developed by Ou et al. [4]: particles were deposited on a TEM grid, then labeled with the intercalating fluorophore deep red anthraquinone number 5 (DRAQ5). The grids were bathed in diaminobenzidine (DAB) and photobleached, causing the release of singlet oxygen and catalyzing the polymerization of DAB localized to the fluorophore. The BCNs were then stained with osmium tetroxide, which has a high binding affinity for DAB.

Figures $1 \mathrm{a}$ and $1 \mathrm{~b}$ show TEM images of combed DNA strands imaged at $100 \mathrm{kV}$. The strands are expected to extend to a maximum of $2.3 \mu \mathrm{m}$ in length $(3.4 \AA$ per base pair); the longest strands in Figures $1 \mathrm{a}$ and $1 \mathrm{~b}$ are $1.7 \mu \mathrm{m}$ and $2.1 \mu \mathrm{m}$, respectively. Figure $1 \mathrm{~b}$ shows a looped strand, a characteristic feature that occurs when both ends of the strand adhere to the surface during combing. Though negatively stained, the negatively charged DNA strands likely interact with the positively charged uranyl species, leading to an effective "positive" staining in which strands appear dark in contrast compared to the background. The width of these strands falls between 5 and $10 \mathrm{~nm}$, slightly larger than reported $2 \mathrm{~nm}$ width of double stranded DNA but potentially broadened by staining effects and microscope resolution. 
Figure 1c shows a representative cryo-TEM image of the unstained cubic network structure of BCNs, and the high angle annular dark field (HAADF) STEM image in Figure 1d demonstrates staining of DNA-loaded BCNs using the ChromEM method. The PPS in the bilayer shell is visible and lower contrast than the stained aqueous channels, indicating that the DNA in the interior channels is stained preferentially to the polymeric materials. The width of this shell in the stained particle (Figure 1d) is 10$13 \mathrm{~nm}$, comparable to the exterior shell width of $12 \mathrm{~nm}$ of the unstained BCN (Figure 1c).

This work demonstrates the application of two systems that can be used to address challenges in soft and biological material imaging through correlative characterization and contrast enhancement. Elongated and aligned DNA provides a platform for correlative methods; ongoing work includes the analysis of DNA by positive staining, fluorescence microscopy, atomic force microscopy, high energy resolution electron energy loss spectroscopy for observation of the phosphor backbone, and imaging by low voltage cryo-TEM, among other techniques. The preparation of BCNs demonstrates targeted staining in nanoscale systems, which contain far lower concentrations of DNA than do cellular systems. We are now able to analyze the distribution of DNA and other macromolecules in nanoparticles where DNA is otherwise indistinguishable from similar or higher contrast components, and we are expanding this method to imaging and analysis of other soft/hybrid nanostructured systems.

\section{References:}

[1] A Bensimon et al., Science 265 (1994), p. 2096-2098

[2] S Bobbala, SD Allen, and EA Scott, Nanoscale 10 (2018), p. 5078-5088.

[3] H Nakao et al., Nano Letters 2 (2002), p. 475-479.

[4] HD Ou et al., Science 357 (2017), p. eaag0025.

[5] This work was supported by Air Force Research Laboratory grant FA8650-15-2-5518 and the National Science Foundation Graduate Research Fellowship Program, and partially supported by Air Force Office of Scientific Research award number FA9550-17-1-0348 and Army Research Office MURI grant W911NF1810200. This work made use of the Electron Probe Instrumentation Center and BioCryo facilities of Northwestern University's NUANCE Center, which has received support from the Soft and Hybrid Nanotechnology Experimental (SHyNE) Resource (NSF ECCS-1542205); the MRSEC program (NSF DMR-1720139) at the Materials Research Center; the International Institute for Nanotechnology (IIN); the Keck Foundation; and the State of Illinois, through the IIN.
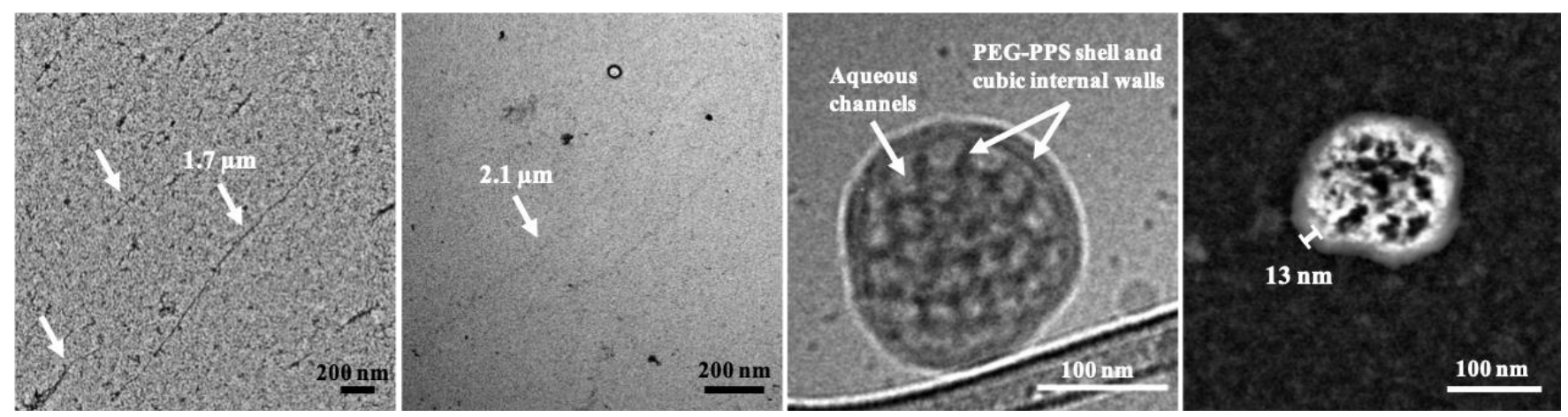

Figure 1. $100 \mathrm{kV}$ TEM images of a) several aligned DNA strands and b) a single looped strand that occurs when both DNA ends adhere before combing, all stained with uranyl acetate. c) Cryo-TEM image of unstained BCN with visible aqueous channels. d) HAADF STEM image of BCN; channels are loaded with DNA and positively stained using the ChromEM method, which targets DNA with the osmium contrast agent. The PEG-PPS shell is visible and lower in contrast than the interior channels. 